\title{
La préhistoire en Vendée de la pierre au bronze
}

\section{Charles-Tanguy Le Roux}

\section{OpenEdition}

\section{Journals}

Édition électronique

URL : http://journals.openedition.org/rao/1421

DOI : $10.4000 /$ rao.1421

ISSN : 1775-3732

\section{Éditeur}

Presses universitaires de Rennes

\section{Édition imprimée}

Date de publication : 31 décembre 2010

Pagination : 221-223

ISBN : 978-2-7535-1383-9

ISSN : 0767-709X

\section{Référence électronique}

Charles-Tanguy Le Roux, «La préhistoire en Vendée de la pierre au bronze », Revue archéologique de l'Ouest [En ligne], 27 | 2010, mis en ligne le 25 février 2012, consulté le 06 décembre 2020. URL : http:// journals.openedition.org/rao/1421 ; DOI : https://doi.org/10.4000/rao.1421

Ce document a été généré automatiquement le 6 décembre 2020

Tous droits réservés 


\title{
La préhistoire en Vendée de la pierre au bronze
}

\author{
Charles-Tanguy Le Roux
}

\section{RÉFÉRENCE}

Joussaume, R., 2009 - La préhistoire en Vendée de la pierre au bronze, La Roche-sur-Yon, édition du CVRH, 192 p. (ISBN 978-2-911253-42-3 ; $25 €$ )

1 La Vendée est un département à l'identité forte mais multiforme, très proche de la Bretagne et de la basse vallée de la Loire au nord mais évoquant déjà le Bassin Aquitain dans sa partie méridionale tandis que, d'est en ouest, on passe des collines des Mauges à une alternance de grandes plages, de falaises basses et de zones marécageuses. Ce faible relief du littoral comme des fonds marins qui le précèdent va de pair avec une grande instabilité du trait de côte en fonction de l'érosion-sédimentation et des variations du niveau de la mer; la carte donnée p. 6 souligne cette composante majeure du paléoenvironnement vendéen au post-glaciaire, composante que l'auteur ne manque pas de souligner car elle a guidé l'activité humaine et largement influencé la conservation des vestiges.

2 Le sous-titre de l'ouvrage (« 4500 ans d'histoire avant les gaulois ») nous précise la fourchette chronologique étudiée - le Néolithique et l'âge du Bronze - et aussi le concept «d'histoire sans écriture » auquel tient Roger Joussaume puisqu'il le reprend en tête de sa conclusion (p. 177). Mais l'auteur sait regarder en amont et en aval sur l'échelle du temps, comme il sait élargir son champ géographique pour éclairer le coeur de son propos. Avant d'être le grand chercheur que l'on connaît, Roger Joussaume fut enseignant; il en a gardé le goût de la pédagogie. On sait aussi que sa carrière scientifique l'a porté en bien des endroits de la planète; il en a gardé le sens de l'universel. L'un et l'autre s'expriment ici avec talent.

3 Le volume s'articule en trois parties. La première (45 p.) s'ouvre sur une présentation quasi-lyrique du propos, suivie de l'attaque quelque peu provocatrice du ch.1: 
«Fouiller [...] à quoi ça sert ? [...] à pas grand'chose... pas plus que le foot, le cinéma, la télévision". Suit une évocation des principales techniques de prospection et de datation (ces dernières hélas quasiment réduites au seul radiocarbone) : dommage que les bonnes vieilles méthodes hypothético-déductives et comparatives passent à la trappe dans un ouvrage destiné à un public déjà sensibilisé, d'autant qu'elles ont largement sous-tendu la mise en place des chronologies présentées au fil des pages qui vont suivre.

Le chapitre 2 (Néolithique et néolithisation) commence par un utile rappel des principaux foyers de primo-domestications (animales et végétales) actuellement reconnus sur la planète pour aboutir à celui qui va nous concerner directement, dans le «croissant fertile» proche-oriental. L'histoire de son émergence à partir du XII ${ }^{e}$ millénaire av. J.-C. est retracée, puis celle de son expansion vers l'ouest jusqu'à ce que ses deux composantes - danubienne et méditerranéenne - séparées par les Alpes depuis les Balkans se retrouvent et s'interpénètrent dans ce qui n'était pas encore la France médiane. Entre les débouchés sur l'océan de la Loire et de la Garonne, on conçoit que l'actuelle Vendée ait eu alors une position-clé que l'auteur nous nous présente dans ses grandes lignes au ch. 3 .

La deuxième partie - Les premiers agriculteurs en Vendée (5 000-2 500 av. J.-C.) représente le coeur de l'ouvrage avec plus de 80 pages. Elle comprend cinq chapitres thématiques (l'habitat, l'équipement matériel, l'économie, la mort, les manifestations symboliques), qui balaient chacune toute la période. Le parti a ses avantages (une bonne fluidité de l'exposé) et ses inconvénients (une relative difficulté à appréhender certains grands sites complexes dont la présentation se retrouve nécessairement désarticulée) ; globalement cependant, l'auteur réussit son pari grâce à un style à la fois fluide et précis. Pour ce qui est de l'habitat, les deux sites majeurs de Machecoul (à l'extrême sud de la Loire-Atlantique) et du Champ-Durand à Nieul-sur-l'Autise forment le corps de l'exposé qui s'achève sur l'espoir de bientôt découvrir en Vendée de grandes maison comparables à celles déjà connues dans les départements voisins (Deux-Sèvres, Vienne, Charente). L'équipement matériel est présenté de manière classique (lithique, osseux, céramique). Pour la première catégorie, un tel ouvrage eût été le lieu idéal pour présenter au public averti mais non spécialiste (qui est clairement visé ici) un tableau synthétique des armatures de flèches de l'Ouest dont les nombreux types sont décrits (mais pas toujours nommés). C'est par contre le cas pour la céramique, sur une double page associant dessins de « vaisseliers » et photographies de « belles pièces ». Quant au bref survol des «parures", il a le mérite de nous rappeler que le concept de «fossile directeur du Néolithique ancien ", aujourd'hui volontiers appliqué systématiquement aux anneaux en pierre, mérite à tout le moins une application raisonnée. Le chapitre sur la vie économique s'ouvre par une présentation des spectaculaires traces de « labours croisés » de Brétignolles-sur-Mer qui, bien que de datation floue (le site sera d'ailleurs évoqué à nouveau à propos du Campaniforme au ch. 9), confirment la production - certes minime par comparaison avec celle des lames de haches - de socs d'araires (rappelons simplement ici l'exemplaire spectaculaire de la Butte-aux-Pierres (Saint-Joachim, Loire-atlantique), en dolérite du «type A » (Le Roux, 1999, p. 154-155). L'environnement calcaire de Champ-Durand, site naguère étudié par l'auteur, y a permis la conservation de restes osseux dont quelques éléments spectaculaires illustrent le discours sur la faune. Enfin, le rôle des termes labiles voire immatériels des 
échanges qui ne manquaient pas de s'établir entre groupes humains est opportunément souligné.

6 Le chapitre 7 ( «La mort au Néolithique ») est bien entendu très largement consacré au riche monumentalisme funéraire vendéen, domaine où l'expérience de R. Joussaume n'est plus à rappeler. Peut-être la part faite aux vieux fantasmes celtomanes pour introduire le sujet y est-elle trop belle ? Aujourd'hui, le thème fait relève heureusement - enfin - de l'histoire des sciences et non plus du débat scientifique, de sorte que sa place eût été plutôt dans un encart : à continuer de le pourfendre ainsi, n'aide-t-on pas plutôt à sa survie? Pour le reste, le tour d'horizon est complet, équilibré et bien illustré ; on regrettera simplement que les coupes de la p 110 ne soient pas repérées sur la carte attenante (un peu petite), alors qu'elles illustrent un thème cher à l'auteur (et bien développé dans les pages suivantes), celui des "territoires mégalithiques». De même, il n'est pas simple de suivre l'exposé relatif à la "Pierre-virante » sur le plan associé (p. 114). Quant au ch. 8 (les manifestations symboliques), il aborde l'autre volet du mégalithisme, celui des pierres dressées, à la lumière des travaux récents de G. Bénéteau qui ont révélé la complexité du phénomène en Vendée (cf. la typologie des "alignements courts", p. 124) et sa relative déconnexion du mégalithisme funéraire alors que la chronologie reste largement en suspens. Le chapitre se termine par une évocation de l'art au Néolithique, dont le tableau reste bien modeste en Vendée si l'on excepte la poterie (figurine d'Auzay, "corne" de St. Florent-des-Bois, pierres à cupules). Dommage que la "Pierre des Farfadets» du Poiré-sur-Vie, bien que longuement décrite, n'ait pas été illustrée; on se consolera avec la spectaculaire photographie du menhir orné de Saint-Macaire-en-Mauges, présenté au prix d'une incursion de quelques kilomètres en territoire angevin!

7 La troisième partie - Les premiers métallurgistes (2 500-800 avant J.-C.) - s'ouvre par un chapitre consacré au "Campaniforme ", bien représenté dans le département, et à ses rapports (pas toujours évidents) avec le Néolithique final d'Artenac. Tout l'âge du Bronze est ensuite évoqué à partir de la documentation vendéenne qui, comme le reconnaît bien volontiers l'auteur, reste globalement assez terne par rapport à ses voisines armoricaine et charentaise malgré quelques découvertes exceptionnelles (planche ouvrée de La Tranche-sur-Mer, enclos funéraire de Longeville-sur-Mer, broche à protomé de cervidé de Challans, roue de Coulon dans les tout proches DeuxSèvres...).

8 Une bibliographie de plus de 70 références termine cet élégant volume au format "hyper-carré » $(23 \times 21 \mathrm{~cm})$, abondamment illustré de photographies en couleurs, plans et dessins (malheureusement non numérotés ni appelés dans le texte ; cela allège certes la présentation d'un ouvrage qui se veut manifestement de " haute divulgation ", mais n'en facilite pas la consultation). Le maquettage est soigné mais - autre reproche prersonnel - le parti de multiplier les documents aboutit à des séries de «figures timbres-poste " pas toujours très lisibles, sed de gustibus et coloribus non disputandum ! Malgré ces petits bémols, Roger Joussaume et le Centre vendéen de Recherches historique peuvent être fiers de nous proposer cet ouvrage très agréable à regarder, facile à lire et riche d'une information factuelle abondante tout comme des pertinentes réflexions personnelles de son auteur. 